\title{
PROBLEMATA ШBOBVHWVLV
}

\section{L'ALBERO DELLA CONOSCENZA: LA DIALETTICA DEL MITO ETERNO DELL'UOMO SECONDO HEGEL}

\section{THE TREE OF KNOWLEDGE: THE DIALECTIC OF THE ETERNAL MYTH OF MAN ACCORDING TO HEGEL}

\author{
Martina Barnabal \\ Recebido em: $11 / 2020$ \\ Aprovado em: 11/2020
}

\begin{abstract}
Il presente contributo si propone di indagare la natura dialettica del mito del peccato originale all'interno del pensiero di Hegel. A scopo introduttivo verrà messo in luce brevemente il procedimento attraverso il quale la filosofia hegeliana opera la traduzione da rappresentazione religiosa a concetto, dimostrando come questa lettura sia alla base dell'interpretazione del mito. In seguito si analizzerà il funzionamento dei movimenti dialettici dell'episodio biblico di Genesi 3 all'interno della Fenomenologia dello Spirito e delle Lezioni di filosofia della religione, per discutere i temi di bene e male, innocenza e imputabilità della colpa, volontà e arbitrio. In questa ricostruzione emergerà l'importanza della dialettica come struttura che permea la coscienza umana così come la realtà in generale. Nel caso specifico dell'albero della conoscenza si assisterà al concretizzarsi di questa eterna conciliazione delle contraddizioni in due ambiti specifici, che verranno trattati nell'ultima sezione: la questione del male da una parte, che verrà dimostrato come momento negativo necessario che innesca il movimento dialettico stesso, e la questione della libertà dall'altra, che apparirà come il risultato dell'emancipazione del soggetto dallo stato naturale in cui si trova nel cosiddetto "giardino degli animali".
\end{abstract}

Parole chiave: Religione; Peccato originale; Male; Libertà; Dialettica

\begin{abstract}
This paper aims to investigate the dialectical nature of the myth of original sin as described by Hegel. For introductory purposes, I will briefly highlight the process by which Hegelian philosophy operates the translation from religious representation to concept, demonstrating how this reading is at the basis of the interpretation of the myth. Then I will analyze the functioning of the dialectical movements of the biblical episode of Genesis 3 within the Phenomenology of Spirit and the Lectures on the Philosophy of Religion, in order to discuss the issues of good and evil, innocence and guilt, will and arbitrariness. In this reconstruction the dialectic will emerge in its importance as a structure that permeates human consciousness as well as reality in general. In the specific case of the tree of knowledge, we will witness the concretization of this eternal conciliation of contradictions in two specific areas, which will be treated in the last section: the question of evil on the one hand, which will be demonstrated as a necessary negative element that triggers the dialectical movement itself, and the question of freedom on the other, which will appear as the result of the emancipation of the subject from the natural state in which he finds himself in the so-called "garden of animals". Keywords: Religion; Original sin; Evil; Freedom; Dialectic
\end{abstract}

\footnotetext{
${ }^{1}$ Dottoranda in Filosofia alla Sapienza Università di Roma.
} 
All'interno della filosofia di Hegel, la vicenda del peccato originale costituisce un nodo di fondamentale interesse. Egli, infatti, riconosce in essa non soltanto una materia religiosa, ma anche l'esposizione dello sviluppo dell'umanità in generale. Come in tutto il resto dei movimenti processuali che costellano il sistema hegeliano, anche in questo caso - e potremmo dire forse massimamente - si assiste al dispiegarsi di una dialettica rigorosa e necessaria che procede per negazioni e conciliazioni. Si tratta di una dialettica, inoltre, strettamente legata all'insorgere dell'autonomia e della libertà, motivo per cui il cosiddetto "mito eterno" si qualifica come una vera e propria storia della maturazione e dell'emancipazione del soggetto. Nelle Lezioni di filosofia della storia Hegel descrive in questo modo l'episodio di Genesi 3:

Vi si narra che l'uomo, creato ad immagine e somiglianza di Dio, avrebbe perduto il suo stato di assoluta soddisfazione per aver mangiato dall'albero della conoscenza del bene e del male. Qui il peccato consiste solo nella conoscenza: questa è l'elemento peccaminoso e per causa sua l'uomo si è giocato la felicità naturale. Solo la coscienza dà luogo alla separazione dell'Io, inteso secondo la sua infinita libertà come arbitrio, dal puro contenuto della volontà, del bene. È una profonda verità: il male risiede nella coscienza; infatti gli animali non sono né buoni né malvagi, e così il semplice uomo naturale. La conoscenza è peccato originale come superamento dell'unità naturale; perciò quel peccato non è una storia accidentale, bensì è la storia eterna dello spirito. Lo stato d'innocenza, questa condizione paradisiaca, è lo stato animale. Il paradiso è un parco, dove possono rimanere solo gli animali, non già l'uomo. L'animale è in unità con Dio, ma solo in sé. Soltanto l'uomo è spirito, ossia è per se stesso. Ma questo essere per sé, questa coscienza sono nel medesimo tempo la separazione dal divino universale. Quando mi mantengo nella mia astratta libertà contro il bene, ecco il punto di vista del male. Perciò il peccato originale è il mito eterno dell'uomo, è il peccato tramite il quale l'uomo si fa uomo (G.W.F. Hegel FilSt, trad. it 2003, p. 269).

Da questo primo estratto possiamo già intuire la moltitudine di temi e questioni che sono in gioco all'interno dell'interpretazione hegeliana: il bene e il male, la coscienza, la libertà, il passaggio da stato naturale a stato spirituale e persino la natura del mito in quanto tale.

A proposito di quest'ultimo argomento, prima di addentrarsi nel vivo del discorso, è bene menzionare qui nell'introduzione un ulteriore movimento dialettico, che è alla base della religione in generale e quindi di tutte le figure del Cristianesimo, compresa quella del peccato originale. Si tratta del ruolo della rappresentazione, ovvero della forma attraverso la quale i concetti vengono veicolati nella sfera della religione. La rappresentazione è un linguaggio particolare a metà tra intuizione e pensiero caratterizzato da una limitatezza di fondo, quella di esporre il contenuto secondo accadimenti casuali inscritti nella successione di spazio e tempo. 
La necessità immanente propria della verità in senso stretto è sacrificata all'altare di una forma sensibile, che pur accennando al contenuto universale del vero continua a servirsi di simboli e immagini accidentali, frutto di un'intelligenza che "è quindi fantasia, immaginazione simbolizzante, allegorizzante o poetante" (G.W.F. Hegel Enc, trad. it. 1996, p. 745). Da ciò segue che le varie determinazioni opposte, che il ragionamento concettuale riesce invece a "tenere insieme", sfociano in contraddizioni insolute, scissioni che la forma religiosa, ancora non speculativa, non è in grado di sanare.

L'atteggiamento qui adottato, dunque, è quello che considera la rappresentazione come il guscio che riveste la verità, la quale, per venire alla luce, deve essere liberata dalle contraddizioni evocate dalla forma sensibile di tale guscio. ${ }^{2}$ Si tratta di un modo di concepire il mito non estraneo alla storia della filosofia, né tantomeno ad alcuni successori di Hegel stesso, famosi per il discorso di "demitologizzazione" legato al passaggio da rappresentazione a concetto. È il caso per esempio di Strauss, anticipatore della sinistra hegeliana, che con la sua Vita di Gesù (1835) produsse la celebre spaccatura tra le due scuole. Nella sua opera, Strauss identifica il mito con la rappresentazione di Hegel e rilegge la storia evangelica alla luce del passaggio critico da forma mitico-rappresentativa e storica delle scritture a concetto della filosofia. Considerando che esiste comunque una differenza tra "storia mitica" dell'Antico Testamento e "storia divina" degli atti e della vita di Cristo, ${ }^{3}$ siamo dell'opinione che entrambe le rappresentazioni svolgono un ruolo fondamentale, da rivalutare a seguito della critica: le immagini e le narrazioni, infatti, una volta intese correttamente, donano "carne" al concetto, lo concretizzano, sono in un rapporto di significazione con esso. Ciò è dovuto in larga parte alla dimensione complessa in cui agisce la rappresentazione stessa, che abbiamo definito un ponte tra intuizione e pensiero. La rappresentazione contiene al suo interno, infatti, entrambi gli elementi: la sensibilità che era propria dell'arte e il pensiero che caratterizzerà invece la filosofia, tanto che queste forme, secondo il manoscritto delle lezioni di filosofia della religione, “finiscono al contempo per mischiarsi” (G.W.F. Hegel FilRel I, trad. it. 2003, p. 193). Ne deriva

\footnotetext{
${ }^{2}$ Ricordiamo che la tesi di fondo di Hegel riguardo a religione e filosofia è che sebbene le forme siano diverse il contenuto rimane lo stesso, la verità del concetto: "Il contenuto è lo stesso, ma, così come Omero dice che alcune cose hanno due nomi - l'uno nel linguaggio degli dei, l'altro nel linguaggio degli uomini ordinari -, allo stesso modo ci sono due linguaggi per quel contenuto" (G.W.F. Hegel Enc, trad. it. 1996, p. 57).

${ }^{3} \mathrm{Hegel}$ stesso si pronuncia in questo senso quando nel corso di lezioni di filosofia della religione del 1827 precisa che il ruolo della storia cambia a seconda della rappresentazione presa in esame: l'albero della conoscenza è appunto un mito ed egli ne critica la storicità come suo difetto formale; la storia presentata nei Vangeli ha invece un'altra valenza, è l'incarnarsi negli eventi particolari di un concetto eterno che necessita di vita, passione e morte, le quali "valgono come qualcosa di pienamente storico" (G.W.F. Hegel FilRel I, trad. it. 2003, p. 335).
} 
di conseguenza che la Vorstellung si presenta come dialettica in se stessa: ${ }^{4}$ il passaggio al pensiero è presupposto già al suo interno così come è presupposta all'interno di quest'ultimo la ripresa che esso opera nei confronti della natura esemplificatrice della rappresentazione. ${ }^{5}$ Non è un caso che ancora oggi si parli di un "ritorno" alla sfera rappresentativa e quindi di un rapporto biunivoco, dialogante, tra rappresentazione e concetto: ${ }^{6}$ la veste sensibile (in questo caso la storia mitica) deve essere dapprima criticata dalla ragione filosofica, che la depura dalle contraddizioni davanti alle quali si ferma la coscienza religiosa non speculativa; in seguito la rappresentazione del mito, subita un'autentica riassegnazione di senso, acquista il ruolo fondamentale di Beispiel, ovvero di "raffigurazione" di quei contenuti veri, propri anche della filosofia, che altrimenti sarebbero abbandonati all'astrattezza delle categorie logiche. ${ }^{7}$

Effettivamente Hegel, mettendo in guardia dal rischio di non riconoscere l'identità di contenuto tra religione e filosofia, aveva sollevato il problema della questione di prospettiva: da un punto di vista non speculativo, come quello del "cattivo intelletto" o della religione ancora immersa nell'universo del nebeneinander e del nacheinander, la rappresentazione assume un significato (un contenuto) diverso ${ }^{8}$ dal punto di vista filosofico, invece, si riconosce quell'identità di contenuto che rende la religione una metafora, un Beispiel appunto, del concetto della filosofia. Questa dinamica tra linguaggio rappresentativo e linguaggio concettuale farà da sfondo alla lettura che Hegel propone del peccato originale e del male: la dialettica vera e propria che li caratterizza può uscire alla luce solo attraverso il ragionamento concettuale della filosofia; considerato invece da un punto di vista meramente rappresentativo e religioso, l'episodio viene frainteso, risultando antitetico nel significato rispetto

\footnotetext{
${ }^{4}$ P. Ricoeur, 1985.

${ }^{5}$ Si pensi per esempio alla rappresentazione per eccellenza, quella costituita dalla persona di Gesù, che si "autoleva" attraverso la morte dell'individuo particolare e sensibile per passare nell'universalità della comunità, la quale allo stesso tempo però riconosce la necessità di un mediatore (una figura) in carne e ossa.

${ }^{6}$ E. Caramelli, 2016; anche lo stesso Hegel accenna a un rapporto di "andata e ritorno" quando afferma, nella recensione agli Aphorismen di Göschel, che "l'autore, quindi, ha toccato un interessante punto di vista, il passare in generale dalla rappresentazione al concetto e dal concetto alla rappresentazione, un passare di qua e di là, che si trova nella meditazione scientifica e da cui si esige qui che sia espresso dappertutto, anche nella rappresentazione scientifica". (G.W.F. Hegel RecG, trad. it. 2001, p. 44).

${ }^{7} \mathrm{Nel}$ corso di lezioni di filosofia della religione del 1824 Hegel elabora ciò che possiamo definire una teoria del significato, in cui si manifesta tutta l'importanza del ruolo "esemplificante" della rappresentazione e dunque la possibilità di un doppio movimento da rappresentazione a concetto e da concetto a rappresentazione (G.W.F. Hegel FilRel I, trad. it. 2003, pp. 93-94); a questo proposito Pierluigi Valenza definisce il movimento come "un movimento nelle due direzioni, dalla rappresentazione al concetto, come significato logico, di pensiero, del contenuto di simboli, immagini, riti dell'universo religioso, e dal concetto alla rappresentazione, trascrizione figurata, simbolica di ciò che è colto in modo disincarnato col pensiero" (P. Valenza, 2017, p. 74).

8 "In tale ambito [quello del cattivo intelletto] Religione e Filosofia sono infatti rappresentate come se l'una escludesse l'altra" (G.W.F. Hegel Enc, trad. it. 1996, p. 59).
} 
all'intendimento che se ne ha tramite la ragione. ${ }^{9}$

Il mito dell'albero della conoscenza, infatti, si presenta agli occhi della coscienza religiosa come un evento dispiegato nella storia e improntato a una spiccata casualità (qualcosa che sarebbe potuto e dovuto non accadere), laddove lo sguardo speculativo riconosce al suo interno quell'incedere inevitabile e necessario che denota tutti i movimenti dialettici. Tuttavia, abbiamo visto che persino i miti come quello qui considerato, che rimane caratterizzato dall'accidentalità della forma rappresentativa e che perciò necessita della critica della filosofia, ${ }^{10}$ possono riguadagnare valore in quanto esposizione metaforica (Beispiel) del vero. Quando si comprende, tramite il ragionamento concettuale, che il mito del peccato originale indica l'inizio della divinizzazione dell'uomo invece che la perdita di uno stato divino, ${ }^{11}$ e si criticano, dunque, i limiti della rappresentazione che hanno fatto sì che esso fosse letto per così dire "al contrario", allora si può iniziare a riconsiderare l'episodio biblico come "il mito eterno dell'uomo, attraverso il quale egli si fa uomo", ovvero come una storia portatrice di grandi significati (contenuti veri!) per il soggetto.

Tenendo presente questo rapporto tra rappresentazione religiosa e concetto della filosofia analizzeremo la trattazione del peccato originale all'interno della Fenomenologia e delle Lezioni di filosofia della religione, concentrandoci inizialmente su una ricostruzione del pensiero di Hegel. In seguito, considereremo più nello specifico i suddetti contenuti veri, che emergeranno dalle dialettiche di bene e male, innocenza e colpevolezza, libertà astratta e concreta, osservate nei primi due paragrafi. In particolare si conviene in questa sede con la lettura che individua una "secolarizzazione" del mito in favore della determinazione del male come negazione necessaria ma soprattutto del peccato e della presa di consapevolezza come momenti fondamentali della Bildung del soggetto libero.

\section{Bene e male nel capitolo VII della Fenomenologia dello Spirito}

La scelta di limitare l'indagine alla Fenomenologia e alle Lezioni di filosofia della religione è dovuta alla maggiore considerazione che Hegel dedica all'argomento in questi

\footnotetext{
${ }^{9}$ Nella seguente analisi osserveremo come i significati attribuiti dalla teologia "classica" a Genesi 3 appaiano diversi se non opposti rispetto a quelli sviluppati dalla filosofia speculativa.

${ }^{10}$ Non è questa ovviamente l'opinione di tutti. Wolf sostiene che il mito sia la forma più adeguata ad esprimere il logos. Egli critica, infatti, Strauss e il progetto di Entmythologisierung e tenta di dimostrare come la narrazione del Sündenfall veicoli significati che la comprensione concettuale ignora o perde durante il processo di traduzione (J. Wolf, 2011).

${ }^{11}$ J. Ringleben, 2005; W. Trillhaas, 1971.
} 
luoghi, in cui ricorrono i significati sopra richiamati del ruolo del male e del negativo da una parte e della costruzione della soggettività e della sua libertà dall'altra. Nella Fenomenologia, infatti, la trasgressione al comandamento divino si declina come un conflitto tra le determinazioni di bene e male all'interno dell'uomo - e specularmente all'interno di Dio ${ }^{12}$ che coinvolge i due temi della necessità del male e dello sviluppo del soggetto. Si parla di uomo e Dio perché questo conflitto fa parte dell'evoluzione della coscienza umana e allo stesso tempo di quell'assoluto che fa da contraltare al "per noi" dell'opera, la quale, come è noto, è appunto il viaggio della coscienza tanto quanto il viaggio dello Spirito che torna a se stesso. Nel capitolo VII, dedicato alla religione, Hegel si appresta a descrivere il movimento del divenire-altro dello Spirito, che dall'unità semplice del primo momento trinitario, la cosiddetta "sostanza nell'elemento del pensiero puro" (G.W.F. Hegel Fen, trad. it. 1995, p. 1005), passa nella sfera della rappresentazione, ovvero di quella realtà concreta che si rivela nella creazione di un mondo e di un uomo.

Sebbene la rappresentazione non sia ancora pensiero concettuale, ${ }^{13}$ essa, senza esserne consapevole, rimane un momento necessario dello sviluppo del concetto, che per essere davvero tale deve uscire da sé e poi riprendersi, ritrovare la sua identità, in questo altro da sé. Nella religione, come si diceva, questo processo si traduce nella celebre figura della creazione del mondo: "Lo Spirito soltanto eterno, cioè astratto, diviene dunque per sé un altro, accede cioè all'esistenza: immediatamente, esso accede all'esistenza immediata. Esso crea dunque un mondo" (G.W.F. Hegel Fen, trad. it 1995, p. 1009). Nell'esteriorità molteplice e "gettata" di questo mondo si distingue poi un "Sé semplice" che è coscienza, spirito esistente, il quale tuttavia non è ancora "per sé", ovvero non è ancora consapevole di essere ciò che è. Si tratta dell'uomo, che per questo motivo può essere definito "innocente" (una determinazione diversa, come vedremo, da quella della bontà!): egli non riflette ancora la razionalità del concetto, non è cosciente della propria negatività interna, non è, in poche parole, autocosciente e pienamente spirituale. Poiché, tuttavia, il procedere dialettico consiste sempre nel non soggiornare in figure statiche, anche nel caso della coscienza singola si verifica una frattura, che si configura come un'introiezione della coscienza stessa in cui essa percepisce il suo essere-altro.

Il divenire-altro di cui Hegel parla è appunto l'insorgere del male: la prima opposizione

\footnotetext{
12 J. Hyppolite, trad. it. 1999.

13 "Essa ha il contenuto senza la sua necessità, e nel regno della coscienza pura, al posto della forma del Concetto, introduce i rapporti naturali di padre e figlio. Poiché anche nel pensiero stesso tale rappresentazione procede rappresentativamente, essa scorge certamente la rivelazione dell'essenza, ma, per via di questa rappresentazione sintetica, vede separarsi l'uno dall'altro i momenti dell'essenza" (G.W.F. Hegel Fen, trad. it. 1995, p. 1007).
} 
che questa coscienza immediata prova entro sé è quella tra bene e male, una distinzione che la porterà a sentirsi estraniata dall'identità immediata dell'innocenza iniziale e quindi da se stessa. Viene fatto accenno diretto in questa sede all'albero della conoscenza, l'immagine principale del mito che stiamo trattando, ma che nella Fenomenologia rimarrà quasi sempre dietro le quinte:

L'uomo viene qui rappresentato nel modo seguente: è accaduto - come qualcosa di non necessario - che egli, cogliendo il frutto dell'albero della conoscenza del Bene e del Male, ha perso la forma dell'uguaglianza con se stesso, ed è stato quindi cacciato dalla condizione della coscienza innocente, dalla natura che si offriva senza esigere lavoro e dal paradiso, giardino degli animali (G.W.F. Hegel Fen, trad. it. 1995, p. 1011).

Emergono una serie di punti, come la natura senza lavoro, la condizione di innocenza o l'animalità, che esamineremo meglio in seguito nella sezione dedicata alle Lezioni, in cui Hegel opera più espressamente la critica e la riassegnazione di senso che abbiamo menzionato in merito ai rapporti tra rappresentazione e concetto.

Intanto possiamo però proseguire il discorso sul bene e il male, facendo notare che essi vengono legati in questo passo della Fenomenologia alla conoscenza, ovvero all'abbandono di quello stato di innocenza iniziale che coincide con la nascita dell'opposizione sopracitata. L'uomo che viene considerato semplice e immediato è l'uomo del giardino che non sente e non vive la frattura e che allo stesso tempo, proprio perché non è mediato dalla scissione, non è consapevole di essere spirito. Nel momento in cui mangia il frutto dell'albero egli produce quell'introiezione "maligna" che lo getta nella diseguaglianza, facendo però così un passo decisivo verso la sua maturazione fenomenologica. Questo divenire-malvagio, infatti, è in realtà l'inizio della conciliazione, che parte dalla presa di coscienza di trovarsi, in quanto spirito naturale e non ancora razionale, in una condizione che in sé è già maligna, poiché non consapevole della propria vera essenza, non ancora "per sé". ${ }^{14}$ A questa presa di coscienza corrisponde la fuoriuscita da tale condizione: la conoscenza del bene e del male è la spinta che fa avanzare il soggetto alla figura successiva.

\footnotetext{
14 "Il Sé deve ritrarsi da tale naturalità e deve introiettarsi, deve, cioè, divenire malvagio. Questo lato, però, è malvagio già in sé: l'introiezione consiste perciò nel convincersi del fatto che l'esistenza naturale è il Male. [...] Questo sapere, dunque, è certamente un divenire-malvagio, ma, tuttavia, è soltanto un divenire del pensiero che pensa il Male: esso è perciò riconosciuto come il primo momento della riconciliazione. Tale sapere, infatti, in quanto ritorno entro sé a partire dall'immediatezza della natura che è determinata come il Male, costituisce a un tempo l'abbandono di questa natura e la morte al peccato" (G.W.F. Hegel, ivi, pp. 1023-1025).
} 
Nell'estratto sopra riportato si precisa, inoltre, come la rappresentazione colga questo passaggio come qualcosa di "non necessario". Il concetto, al contrario, essendo caratterizzato lui stesso dalla necessità, pone il male come momento di negazione necessaria nella dialettica che porta alla conciliazione concreta, frutto della mediazione costituita appunto dalla distinzione in se stessi.

Altro limite della forma rappresentativa è quello di concepire bene e male come esteriori l'un l'altro, proprio per quell'incapacità di "tenere insieme" le determinazioni a cui si accennava nell'introduzione. Il risultato è che l'uomo si trova in un'opposizione insoluta che qualifica lui stesso come il male, mentre proietta al di fuori, in Dio, la coscienza del bene. La stessa situazione, come fa notare Hyppolite, si verifica specularmente in Dio stesso, il quale non riconosce il momento del divenire-altro come una negazione avvenuta al suo interno e rimane avviluppato nelle rappresentazioni della caduta del "figlio primogenito della luce" (Lucifero) e della generazione del figlio unigenito, tutti accadimenti "negativi" esterni all'essenza divina. ${ }^{15}$ In entrambi i casi, tuttavia, la negazione è immanente e l'essere-altro dell'opposizione viene ricompreso come proprio momento.

Parallelamente, nel fatto che l'essenza divina esteriorizzi se stessa fin dall'inizio e che la sua esistenza si introietti e divenga malvagia, è non esplicito ma implicito che, in sé, questa esistenza malvagia non è estranea all'essenza divina. L'essenza assoluta sarebbe soltanto una parola vuota se ci fosse veramente un altro da essa, se ci fosse una caduta da essa; il momento dell'essere-entro-sé, invece, costituisce il momento essenziale del Sé dello Spirito (G.W.F. Hegel Fen, trad. it. 1995, pp. 1017-1019).

La risoluzione di questa doppia opposizione si presenta nell'individuo che in carne e ossa rivela l'unità di umano e divino, essere e essere-altro. Si tratta della persona di Gesù, che nella Fenomenologia non viene nominata espressamente, ma che comunque è sottointesa alla "riconciliazione dell'essenza divina con l'altro in generale, e precisamente con il pensiero di questo altro, con il Male" (G.W.F. Hegel Fen, trad. it. 1995, p. 1019).

La natura di questo momento fondamentale, che appare anche alla coscienza rappresentativa e che dunque dovrà a sua volta essere spogliato dalle contraddizioni di quest'ultima, ${ }^{16}$ necessiterebbe di una trattazione a sé stante ancora più ampia. Per questo motivo

\footnotetext{
${ }^{15}$ G.W.F. Hegel, ivi, p. 1011.

16 C'è bisogno di un'ulteriore negazione affinché l'identità tra umano e divino venga interiorizzata dall'autocoscienza, quella della morte del mediatore che innesca il passaggio alla comunità, in cui la rappresentazione sensibile dell'individuo particolare dilegua: "Una volta rimossa, la presenza immediata dell'essenza autocosciente è l'essenza stessa come autocoscienza universale” (G.W.F. Hegel, ivi, p. 1017).
} 
ci limiteremo a sottolineare come nella Fenomenologia il momento negativo rappresentato dall'insorgere del male nella coscienza umana e nello sviluppo di Dio sia la premessa necessaria da cui scaturisce la conciliazione che si ha in Cristo e in seguito nella comunità. La conoscenza che spezza l'unità originaria della coscienza con se stessa eleva quest'ultima a uno stadio sì di scissione, ma allo stesso tempo di consapevolezza dell'esistenza di un altro da sé. Solo a partire da questa condizione può essere ripristinata l'unità con l'esteriorità: un'unità questa volta concreta, che contiene il male al suo interno come momento necessario alla dialettica del suo sviluppo.

Parlando nel linguaggio immaginifico della rappresentazione, e restituendogli il significato della sua verità concettuale, bisogna necessariamente cogliere il frutto dell'albero della conoscenza, uscire dal "giardino degli animali", violare quello che solo per la coscienza religiosa è un vero divieto. Si tratta di una necessità dovuta alla natura logica del concetto, ovvero di quella struttura che soggiace all'ontologia del reale, che come si è già ribadito consiste in un fuoriuscire dalla propria identità semplice, generando una scissione, per poi tornare in sé conservando quest'ultima come proprio momento. Tale necessità caratterizza anche il cammino fenomenologico della coscienza, la quale giunge a costituirsi come soggetto spirituale e divino, e quindi, come vedremo, libero, perché contiene la negazione al suo interno. Il peccato, di conseguenza, non si configura come una trasgressione che l'uomo arbitrariamente avrebbe potuto evitare, bensì come la chiave di volta del suo processo di formazione.

\section{Lezioni di filosofia della religione: storia dell'abbandono del giardino zoologico}

Come è noto, tra il 1821 e il 1831 Hegel tenne dei corsi di filosofia della religione all'università di Berlino, dei quali ci pervengono un manoscritto del 1821 e gli appunti dei corsi del 1824 e del 1827 (oltre a qualche annotazione risalente al corso del 1831, di cui tuttavia non abbiamo altre notizie). Ricostruire uno sguardo unitario sulle lezioni non è un'impresa facile, dal momento che lo stesso maestro modifica diverse delle sue posizioni durante gli anni. Abbiamo cercato e cercheremo, tuttavia, di restituirne un quadro organico, facendo attenzione a specificare la provenienza (corsi o manoscritto) delle singole citazioni.

Il punto di partenza comune è che l'uomo, nel suo stato naturale e dunque nella sua immediatezza, non è come deve essere. Nel manoscritto si legge: "lo spirito naturale è essenzialmente ciò che egli [l'uomo] non deve essere e rimanere” (G.W.F. Hegel FilRel III, trad. it. 2011, p. 54); nel corso del 1824: “l'uomo, come uomo immediatamente naturale, deve 
considerarsi come un uomo che è come non deve essere" (G.W.F. Hegel FilRel III, trad. it. 2011, p. 149); infine nel 1827: "se l'uomo è soltanto secondo natura, allora è malvagio" (G.W.F. Hegel FilRel III, trad. it. 2011, p. 231). In quest'ultimo corso si fa più espressamente riferimento alla confusione che le determinazioni "buono per natura" e "malvagio per natura" generano nella filosofia e nel pensiero comune, ma anche negli altri appunti emerge chiaramente la questione dell'essenza dell'umanità così come essa appare immediatamente.

Come nella Fenomenologia, c'è dunque un problema legato al male e a ciò che qui viene definito spirito naturale: rimanendo nell'innocenza dell'esistenza immediata si sosta in uno stato incompleto, che appare come malvagio, poiché non si riconosce la propria vera essenza spirituale, che abbiamo visto consistere nella struttura processuale della ragione e del concetto; dal momento che, tuttavia, quest'ultima è l'in-sé del soggetto, si afferma talvolta che l'uomo è invece buono per natura, spirito in sé per l'appunto. Il problema, secondo Hegel, è posto in maniera sbagliata poiché lo stato di innocenza pre-razionale in cui l'uomo si trova non gli permette di essere consapevolmente buono o cattivo: l'uomo immediato viene, infatti, paragonato ai bambini che non hanno ancora sviluppato la componente razionale e spirituale; ${ }^{17}$ questa componente, al contrario, prevede che egli sia imputabile di colpa, conscio della sua volontà. $^{18}$

Affinché l'in-sé dello spirito venga espresso nella realtà effettuale, si deve verificare un passaggio, un salto dialettico che emancipi l'individuo dalla condizione di innocenza "zoologica" e di "volontà naturale". Sia nel manoscritto che negli appunti dei corsi Hegel conviene, infatti, che l'uomo nel suo stato di natura non vive nella volontà concreta, bensì in un egoismo caratterizzato dalla brama e dalle inclinazioni dell'animalità. Così come la coscienza della Fenomenologia doveva introiettarsi in se stessa per rompere l'identità immediata con sé, anche in questo caso l'uomo deve fuoriuscire dalla naturalità per giungere alla conciliazione della volontà razionale, quella che riconosce le leggi, l'universale. ${ }^{19}$

\footnotetext{
${ }^{17} \mathrm{Dal}$ corso del 1824: "I bambini non sono malvagi, e quella determinazione sembra non essere adeguata neanche rispetto a molti popoli e individui. I bambini sono piuttosto innocenti, e precisamente perché non hanno alcuna volontà, non hanno ancora la capacità di intendere e di volere, mentre la malvagità implica il poter prendere decisioni, l'avere una volontà, l'avere un'intellezione della natura delle azioni' (G.W.F. Hegel FilRel III, trad. it. 2011, p. 150).

${ }^{18}$ Dal corso del 1827: "L'uomo non deve essere innocente, non deve essere un animale; dato che è buono, non deve esserlo nel senso in cui è buona una cosa naturale, bensì deve riconoscere la sua colpa, la sua volontà, deve essere imputabile. Colpa significa imputabilità" (G.W.F. Hegel, ivi, p. 232).

${ }^{19} \mathrm{Dal}$ corso del 1824: "La volontà naturale è la volontà della brama, dell'inclinazione, che vuole l'immediato, che non vuole ancora questo; infatti, perché essa sia volontà razionale, c'è bisogno di una coscienza che abbia in generale un sapere dell'universale e che comprenda che la legge è l'elemento razionale. E' la richiesta rivolta all'uomo, di non essere volontà naturale, di non essere come egli è solo per natura" (G.W.F. Hegel, ivi, p. 151).
} 
Come vedremo nell'ultima sezione, nell'acquisizione di una simile volontà si assiste alla maturazione che porta il soggetto ad essere libero nel senso concreto e hegeliano del termine. Proprio questo passaggio, però, evoca il male, che è appunto la conoscenza stessa, responsabile di ciò che possiamo definire ora come arbitrio, la capacità di scegliere tra bene $\mathrm{e}$ male di cui sono sprovvisti gli animali. L'arbitrio, essendo consapevole della contraddizione tra queste due determinazioni, si configura come una scissione che conduce l'uomo in uno stato di estraneazione dalla natura e da se stesso: egli ha rotto l'identità immediata dell'innocenza infantile, provocando un dolore apparentemente insolvibile. ${ }^{20}$ La dialettica, tuttavia, come abbiamo già avuto modo di constatare, consiste nella conciliazione di quelle contraddizioni che hanno innescato i processi: la conoscenza, infatti, si comporta come pharmakon che contemporaneamente induce la malattia e la cura, allontana l'uomo dalla sua condizione paradisiaca iniziale, gettandolo nella disuguaglianza, e allo stesso tempo lo eleva alla spiritualità implicita nel suo essere soggetto divino. ${ }^{21}$

Questo procedere logico, che si rivela agli occhi del concetto come qualcosa di necessario, viene descritto in maniera figurata dalla coscienza religiosa. La forma rappresentativa, infatti, manifesta questo passaggio da stato di innocenza a stato di conoscenza e imputabilità della colpa come una storia narrata, un insieme di immagini sensibili che vanno a costituire un vero e proprio mito. Le due condizioni di paradiso e caduta, tuttavia, risultano immediatamente esteriori: esiste tra di esse una connessione, ma solo nel senso di un legame casuale, in cui gli eventi si verificano senza essere compresi concettualmente (e quindi senza essere necessari). ${ }^{22}$ Abbiamo qui lo scontro tra rappresentazione e concetto che ha fatto da sfondo a tutti i procedimenti finora indagati: bisognerà analizzare il mito per spogliarlo dalle contraddizioni della sua veste sensibile e riqualificare tale veste attraverso la riassegnazione di significato dell'interpretazione speculativa. Questa è la descrizione che Hegel fornisce della rappresentazione mitica, riferendosi esplicitamente alle scritture (conviene riportare qui l'intero

\footnotetext{
${ }^{20}$ Dal corso del 1827: "Di fatto ciò significa che l'uomo si è innalzato alla conoscenza del bene e del male, e questa conoscenza, questa distinzione è la fonte del male, il male stesso. La malvagità viene posta nell'atto del conoscere, nella coscienza. [...] Infatti conoscere, coscienza in generale, equivale a giudicare, a distinguersi in-se-stesso" (G.W.F. Hegel, ivi, pp. 233-234).

${ }^{21}$ Dal corso del 1824: "Lo spirito deve considerare se stesso, e in questo modo c'è la scissione; egli non deve restare fermo a questo punto di vista, al fatto che esso non è come in sé; egli deve diventare adeguato al suo concetto, allo spirito assoluto. Dal punto di vista della scissione questo è anzitutto un altro, ed esso stesso è anzitutto volontà naturale; esso è scisso in-sé; e quindi questa scissione è il suo sentimento o la sua coscienza della contraddizione, ed è con ciò posto il bisogno del toglimento della contraddizione, la conciliazione" (G.W.F. Hegel, ivi, p. 156).

${ }^{22}$ Dal manoscritto: "è stato ascritto dapprima alla rappresentazione il fatto che la connessione venga rappresentata come un passaggio tra due condizioni. Ciò che è proprio della rappresentazione come tale, è solo che sono due condizioni tra loro connesse, non che quella immediata è essa stessa un passaggio" (G.W.F. Hegel, ivi, p. 61).
} 
passo del corso del 1827, per poter meglio mettere in luce le contraddizioni che derivano dalla natura rappresentativa):

\begin{abstract}
Questa è quindi la guisa figurata in cui questa determinazione concettuale si manifesta nella rappresentazione come una storia, essa è rappresentata per la coscienza in una guisa intuitiva, sensibile, tanto da venir considerata come qualcosa di accaduto. È la famosa storia raccontata nella Genesi. Si dice che Dio abbia creato l'uomo a sua immagine: questo è il concetto dell'uomo. L'uomo viveva nel paradiso; lo si può chiamare un giardino zoologico. Questa vita viene definita la condizione dell'innocenza. Si dice inoltre che nel paradiso vi fosse l'albero della conoscenza del bene e del male, e che l'uomo ne abbia mangiato i frutti contravvenendo al comandamento di Dio. Da un lato si afferma formalmente che questo atto di mangiare è stato la trasgressione di un comandamento. Ma l'essenziale è il contenuto, cui bisogna tener fermo, e cioè che il peccato sta nel fatto che l'uomo ha mangiato dall'albero della conoscenza del bene e del male, e qui compare anche la finzione del serpente, pronto a insinuare che l'uomo sarebbe diventato uguale a Dio, se avesse avuto la conoscenza del bene e del male (G.W.F. Hegel FilRel III, trad. it. 2011, p. 233).
\end{abstract}

Se osserviamo ora tale rappresentazione attraverso la lente del concetto, risulterà chiaro come emergano contenuti antitetici rispetto a quelli osservati dalla coscienza religiosa. Si parla giustamente di un'umanità creata a immagine e somiglianza di Dio: tale immagine si riflette nel fatto sopra richiamato che lo spirito è già nell'immediatezza naturale dell'uomo, sebbene ancora in versione inespressa.

Il problema nasce, secondo Hegel, nel momento in cui la rappresentazione non comprende quel movimento dialettico interno per il quale l'innocenza iniziale dell'uomobambino deve essere superata tramite la scissione del conoscere, la consapevolezza del male e l'imputabilità della colpa. La coscienza religiosa, infatti, pur manifestando tale passaggio attraverso le immagini mitiche del giardino e dell'albero della conoscenza, non ha ancora la capacità di ragionare speculativamente, di reggere il "travaglio del negativo", e concepisce l'acquisizione della conoscenza e dell'arbitrio come la trasgressione di un divieto, come una caduta da quello stato paradisiaco di iustitia originalis che bisognava invece mantenere. Effettivamente, come si diceva, la conoscenza del bene e del male è essa stessa il male (l'estraneazione dell'uomo dalla natura e da se stesso), ma è quel male che, come nella Fenomenologia, innesca il rovesciamento nella conciliazione, è il male che nel momento in cui si pone dilegua in favore dell'elevazione al divino e allo spirituale. ${ }^{23}$

\footnotetext{
${ }^{23}$ Dal corso del 1824: "La conoscenza è il principio della spiritualità, che però, come ho detto, è anche il principio della guarigione dal danno della separazione. In questo principio della conoscenza è posto anche il principio della
} 
A questo proposito, anche nella figura del serpente si riconosce un'incomprensione, dovuta alla forma rappresentativa, a cui si sostituisce una nuova lettura: la promessa di diventare divini, infatti, non era affatto una menzogna, tanto che Dio stesso conferma che Adamo è diventato come lui. ${ }^{24}$

Segue un'altra serie di questioni legate all'episodio della cacciata del paradiso, che vengono fraintese dalla rappresentazione e che ricevono nuovi significati come nei casi che abbiamo appena esposto: 1'ereditarietà del peccato, le pene naturali (il lavoro in particolare), la mortalità dovuta all'allontanamento dall'albero della vita, sono tutti riletti dal concetto non come punizioni, ma come tratti definitori e necessari del soggetto umano, che deve appunto lavorare per distinguersi dall'animale e soprattutto morire per realizzare la sua natura di individuo finito. ${ }^{25}$ Poiché poi il dolore provocato dalla scissione sfocia anche in questa situazione nella proiezione del bene al di fuori dell'uomo, in un Dio "separato", la veste della conciliazione si troverà nuovamente in Cristo, l'uomo-Dio che riunisce l'umanità dannata e il Dio trascendente, la rappresentazione della verità logica per cui finito e infinito si rapportano in un'identità mediata e concreta. ${ }^{26}$ Nei corsi di lezioni si assiste, dunque, allo svolgimento di quella dialettica che avevamo già osservato nella Fenomenologia: attraverso le immagini della Genesi, criticate e riproposte dalla filosofia speculativa, vengono esplicate, come veri nuclei di significato della rappresentazione, la natura del male e la formazione dell'individuo come soggetto spirituale, in questo caso con un maggiore accento sulla volontà e l'arbitrio, che osserveremo essere di centrale importanza nello sviluppo del concetto di libertà.

divinità che, attraverso un bilanciamento futuro, deve arrivare alla sua conciliazione, alla sua verità" (G.W.F. Hegel, ivi, p. 154).

${ }^{24} \mathrm{Dal}$ corso del 1827: "Quando il racconto riporta che un essere estraneo, il serpente, avrebbe traviato l'uomo, facendogli credere che sarebbe diventato uguale a Dio se solo avesse saputo distinguere il bene dal male, ci si immagina che l'azione dell'uomo sia scaturita dal principio malvagio. Ma si mette in bocca a Dio stesso la legittimazione che la conoscenza del bene e del male rientra nell'essenza divina dell'uomo. Egli stesso dice: «Vedi, Adamo è diventato come uno di noi». Le parole del serpente non erano quindi affatto un inganno. Questo particolare viene di solito trascurato a causa del pregiudizio che si ha; si pensa che sia un'ironia di Dio, che Dio abbia fatto della satira" (G.W.F. Hegel, ivi, p. 235).

${ }^{25}$ Dal corso del 1824: "Ci si immagina inoltre che l'uomo abbia ricevuto delle pene naturali, dei mali naturali; questo è un contenuto incerto, ma in effetti il lavoro di Adamo è una conseguenza della conoscenza; gli animali non lavorano; è al contempo l'impronta della superiore natura spirituale dell'uomo. Si dice inoltre che Adamo ed Eva sarebbero stati cacciati dal paradiso, per non assaggiare anche dell'albero della vita. In ciò è insito il fatto che gli individui arrivano alla conoscenza, ma l'individuo resta un singolo e quindi un individuo mortale" (G.W.F. Hegel, ivi, pp. 154-155).

${ }^{26}$ Dal corso del 1827: "Il concetto delle religioni precedenti si è purificato fino ad arrivare a questa opposizione, e, dato che questa opposizione si è mostrata e si è presentata come bisogno effettivo, ciò è stato espresso in questi termini: «Ma dato che il tempo era compiuto, Dio inviò suo figlio». Ciò significa: è presente lo spirito, il bisogno dello spirito che mostra la conciliazione" (G.W.F. Hegel, ivi, p. 241). 


\section{Il male necessario e la libertà concreta dell'individuo}

Il presente contributo non pretende di affrontare questi argomenti nella loro interezza, ma mira semplicemente a mettere in luce il loro legame con il mito del peccato originale così come risultato dalla trattazione che abbiamo riportato della Fenomenologia e delle Lezioni. Per quanto riguarda il tema del male, l'esposizione della dialettica interna alla coscienza e a Dio stesso, che abbiamo portato avanti nel paragrafo sulla Fenomenologia, dimostra come il momento negativo del divenire-malvagio sia parte integrante del processo di sviluppo dello spirito. Questo doppio movimento, che si risolve infine nell'identità tra Dio e uomo che è al centro della filosofia della religione di Hegel, ricorre anche nelle sue Lezioni. Negli appunti del corso del 1827 troviamo riassunta la questione nella seguente maniera:

Il male, uno dei due lati, è stato determinato astrattamente come soltanto l'altro, il finito, il negativo, e Dio è stato posto dall'altro lato come il bene, il positivo, il vero. Questa è una rappresentazione non-vera. Ma quel negativo, quell'altro contiene in se stesso anche l'affermazione, e deve giungere alla coscienza che nel negativo è contenuto il principio dell'affermazione, che in quest'ultimo si trova il principio dell'identità con l'altro lato, allo stesso modo in cui Dio, in quanto è il vero, non è soltanto l'astratta identità con sé, bensì l'altro, la negazione, il porsi altrimenti è la sua propria determinazione essenziale, la determinazione propria dello spirito (G.W.F. Hegel FilRel III, trad. it. 2011, p. 243).

Il risultato è che il male non è un elemento "esteriore", non vive separato e anteposto come un'autonomia a sé stante: il male, in Hegel, non è fuori di Dio come in un Lucifero caduto né fuori dall'uomo come in un serpente che seduce e inganna. Quest'ultimo non è il principio della malvagità, non apre ad alcuna satanologia, ${ }^{27}$ e non esistono in altri luoghi riferimenti al diavolo come causa ed essenza della depravazione morale. ${ }^{28}$ Tantomeno avremo una situazione in cui Dio e uomo, l'uno completamente trascendente rispetto all'altro, contengono tutto il bene da una parte e tutto il male dall'altra. Il finito, infatti, va incontro alla conciliazione con l'infinito proprio perché entrambi vivono la scissione costituita dal momento negativo del male, che dunque si configura come necessario.

La necessità è un'altra grande caratteristica, insieme a quella dell'immanenza, della dialettica tra bene e male, che come abbiamo visto rispecchia il movimento logico del concetto e quindi la processualità stringente con cui si squaderna il vero. Nel caso del peccato originale,

\footnotetext{
${ }^{27}$ W. Trillhaas, 1971.
}

${ }^{28}$ V. Mancuso, 1996. 
l'uomo deve conoscere e provare l'isolamento del suo essere-altro rispetto allo stato naturale in cui si trovava, deve dunque cadere nel male e nella contraddizione del conoscere, i quali a loro volta saranno il motore della sua guarigione, "la sorgente della conciliazione infinita" (G.W.F. Hegel FilSt, trad. it 2003, p. 269). Il fatto che la questione ci sia presentata come contingente ed esteriore, esattamente il contrario della necessità e dell'immanenza appena citate, è dovuto ai limiti della rappresentazione di cui ci siamo già occupati. Hegel scrive che a causa della forma rappresentativa con cui la religione veicola il contenuto vero (quel contenuto che è identico al concetto della filosofia) "ciò che è interiore è espresso come esteriore, ciò che è necessario come contingente" (G.W.F. Hegel FilRel II, trad. it. 2009, p. 281).

Immanenza e necessità, d'altronde, sono le categorie con cui Hegel considera i momenti critico-negativi di tutti i processi logici: anche all'interno della dialettica tra vero e falso, che viene discussa nella Prefazione della Fenomenologia, quest'ultimo assume il ruolo di elemento contraddittorio e quindi manchevole, ma allo stesso tempo necessario e ineludibile per il raggiungimento della verità concreta. ${ }^{29}$ In questo caso Hegel lega espressamente la questione proprio a quella del male:

\footnotetext{
Il vero e il falso appartengono ordinariamente alla categoria di quei pensieri determinati che vengono considerati privi di movimento e come essenze particolari che stanno l'una di fronte all'altra, ciascuna in un rigido isolamento e senza alcun punto in comune con l'altra. Contro una tale concezione, invece, bisogna affermare che la verità non è affatto una moneta coniata che, così com'è, sia pronta per essere spesa e incassata. C'è un falso tanto poco quanto c'è un male. Il male e il falso non hanno affatto la malvagità del diavolo, e a prenderli per entità diaboliche si finisce col farne dei soggetti particolari. Il falso e il male, invece, sono soltanto degli universali, anche se si tratta di due essenzialità distinte (G.W.F. Hegel Fen, trad. it. 1995, p. 93).
}

Ma il male, dunque, è sempre giustificato? Tornando al mito del peccato originale, accenniamo qui a questa problematica interessante, consci che lo statuto del male all'interno della filosofia hegeliana costituisce la fonte di tutta una serie di difficoltà che non possiamo certo risolvere in queste sede: se il male, infatti, c'è ed è sempre necessario allo svolgimento dialettico si rischia di cadere in un giustificazionismo pericoloso. Come è già stato osservato da altri interpreti, ${ }^{30}$ il male che Hegel considera nella spiegazione di Genesi 3 ha due forme principali: quello della scissione costituita dalla conoscenza, che genera dapprima l'insorgere dell'arbitrio e porta in seguito alla conciliazione, e quello costituito invece dalla naturalità dello

\footnotetext{
${ }^{29}$ M. Bordignon, 2016.

${ }^{30}$ J. Ringleben, 2005; W. Trillhaas, 1971.
} 
stato originario. Mentre il primo è appunto necessario, perché innesca il procedimento dialettico e quindi, in ultima istanza, dilegua (motivo per cui i peccatori vengono "perdonati" e nella stessa liturgia si parla di felix culpa), il secondo è caratterizzato da un'animalità che rimane cristallizzata in una finta positività (l'identità astratta e immediata tra uomo e natura).

Potremmo dire con Trillhaas che questa seconda tipologia di male è quella che si verificherebbe qualora l'uomo non mangiasse dall'albero della conoscenza del bene e del male, qualora la volontà naturale del singolo venisse anteposta alla legge razionale dello spirito e non si giungesse mai alla risoluzione dialettica. In questo perdurare della condizione originale, al contrario che nel male costituito dalla conoscenza e dall'arbitrio, non ci sarebbe alcuna giustificazione. Potremmo anche obbiettare, tuttavia, che nella filosofia hegeliana non se ne correrebbe nemmeno il rischio, dal momento che l'intera contraddizione è già risolta, la dialettica è necessariamente già da sempre e per sempre innescata, quella volontà astratta $\mathrm{e}$ "maligna" è già in un certo senso abbandonata. Secondo Hegel, tra l'altro, sarebbe comunque un errore definirla tale perché essa è inconsapevole, non è volontà vera capace di commettere il vero male (o il vero bene). ${ }^{31}$ In ogni caso risulta chiaro come la questione sia appunto legata alla natura della volontà e dell'arbitrio, temi che troviamo in stretta relazione con quello dello sviluppo della coscienza e della libertà del soggetto. La fuoriuscita, infatti, da quello stato di natura rappresentato dal giardino degli animali in cui regnano brama e inclinazioni (seconda tipologia di male), coincide con il momento negativo dell'arbitrio (prima tipologia di male), che porta infine alla conciliazione in una volontà concreta, ovvero razionale. In questa condizione il soggetto è libero.

Ci troviamo d'accordo con l'interpretazione di Ringleben per quanto riguarda l'intera tematica, da lui trattata ampiamente come significato principale del mito di Genesi $3 .{ }^{32}$ Lontani quindi dalla critica di Kierkegaard per cui Hegel sarebbe un filosofo della necessità logicometafisica che opprime i singoli soggetti, mettiamo in risalto la seconda grande questione del mito, lo sviluppo della coscienza individuale e della sua libertà.

Per comprendere quale sia il legame tra l'albero della conoscenza e la libertà del soggetto bisogna innanzitutto specificare che cosa Hegel intenda per libertà. Quest'ultima risulta da sempre al centro del suo pensiero e potrebbe essere indagata da più punti di vista, a partire da quello logico per finire con quello socio-politico. La libertà, infatti, si configura

\footnotetext{
${ }^{31}$ Wolf parla addirittura di un male pre-convenzionale, che determina Adamo ed Eva come animali inconsapevoli delle loro azioni (si tratta davvero allora di un male?), e male post-convenzionale, che segue il peccato dell'albero della conoscenza (J. Wolf, 2011).

32 J. Ringleben, 1977.
} 
all'interno della filosofia hegeliana come il carattere principale dell'assoluto e quindi dell'interezza del reale, natura compresa. ${ }^{33}$ Essa coincide con il movimento dialettico del concetto che abbiamo già indagato, si trova perciò ad essere la verità della necessità: "Nel concetto si è quindi aperto il regno della libertà" (G.W.F. Hegel Log II, trad. it. 1981, p. 657).

In termini più pratici, e dunque anche etici, la libertà si costruisce come un movimento di sintesi in cui gli interessi particolari dei singoli, invece di rimanere soggettivi o arbitrari, si trovano in identità con l'interesse universale della ragione e del vero: "La libertà vera è, in quanto eticità, questo: che il volere non ha per suoi scopi un contenuto soggettivo, cioè egoistico, ma un contenuto universale" (G.W.F. Hegel Enc, trad. it. 1996, p. 463). In questo caso specifico è importante determinare che cosa sia la libertà rispetto alla natura, ovvero a quello stato innocente di cui parla il mito.

A un primo sguardo risulterà che per Hegel essere liberi significhi emanciparsi dalla brama e dalle passioni della nostra parte più "animale". Effettivamente non si è in errore, ma bisogna determinare meglio la questione per non cadere nel malinteso di uno Hegel nemico della naturalità o dei sensi. Come abbiamo già osservato, la natura concepita in maniera immediata, quella natura in cui si trovano Adamo ed Eva prima di mangiare dall'albero della conoscenza, non corrisponde alla natura vera dell'uomo, la quale, essendo spirituale, si conquista come risultato di un processo di mediazione (innescato dalla trasgressione al comandamento divino).

Nella prima situazione incontriamo la cosiddetta "volontà naturale", che non è volontà concreta, e quindi vera libertà, perché non è razionale. La volontà naturale, infatti, non viene diretta dalle leggi universali della ragione, da quella struttura ovvero che permea Dio, l'uomo e il mondo e che non può fermarsi davanti alle posizioni meramente soggettive e particolari. Essa non corrisponde, in altre parole, alla libertà intesa come caratteristica principale della totalità. Poiché, tuttavia, quest'ultima comprende ovviamente anche la natura, persino questa sfera, come vedremo, risulterà in realtà libera (evidentemente, però, non in quella forma immediata in cui si esercita l'egoismo degli impulsi!). A causa di questo carattere immediato e irrazionale, infatti, si dice che l'uomo immediatamente non è come deve essere, anzi è malvagio per natura secondo quella malvagità egoista, ma inconsapevole, che abbiamo visto essere uno dei due intendimenti del male (il secondo). ${ }^{34}$

\footnotetext{
${ }^{33}$ L. Cortella, 2011

${ }^{34}$ Dal corso del 1827: "La richiesta assoluta è che l'uomo non perseveri come volontà naturale, come essere naturale. Egli è, sì, al contempo coscienza, ma, come uomo, può pur essere un essere naturale, nella misura in cui l'elemento naturale costituisce lo scopo, il contenuto, la determinazione del suo volere. Occorre considerare più
} 
Nella seconda situazione, invece, quella della vera natura dell'uomo, in cui si apre la volontà concreta, si assiste ad un'adequatio a quella ragione universale che determina la spiritualità dell'individuo, ovvero il suo essere divino e in ultima analisi libero. Questo risultato è il frutto del divenire-malvagio (secondo il primo intendimento osservato in Trillhaas) che, seguendo la tripartizione della Filosofia del diritto tra volontà naturale, riflettente e libera in sé e per sé, potremmo qualificare come il secondo di questi momenti, in cui insorge l'arbitrio, la capacità di scegliere. Esso, tuttavia, è a sua volta diretto dagli impulsi particolari del "questo" o del "quello", è un momento di scissione che si trova in costante contraddizione con l'universalità. ${ }^{35}$ Tale insorgere del male è stato però dimostrato come farmaco che scatena la malattia e cura al contempo, motivo per cui esso viene rappresentato con la figura della trasgressione e successiva caduta dei primi uomini, che concettualmente coincide al contrario con l'inizio della loro liberazione e divinizzazione. ${ }^{36}$

Come si diceva, l'obbiettivo principale della filosofia della religione di Hegel è, dopotutto, la rivelazione dell'identità tra la natura umana e quella divina. ${ }^{37}$ Nelle sue parole essere divini significa essere razionali e l'uomo, in quanto spirito in sé in grado di esserlo anche per sé, è destinato per sua propria costituzione a diventare tale. Mentre la natura, infatti, pur condividendo anch'essa l'impianto razionale e spirituale che caratterizza l'interezza del reale, non può pervenire all'autocoscienza, alla consapevolezza di tale impianto, e viene definita per questo una "contraddizione irrisolta", 38 si dice che l'uomo sia creato a immagine e somiglianza di Dio proprio perché può uscire da questa contraddizione, può compiere quel cammino fenomenologico e ontologico che lo porta ad essere spirito in sé e per sé. Razionale, divino,

\footnotetext{
da vicino questa determinazione: l'uomo è uomo in quanto soggetto, e, in quanto soggetto naturale, egli è questo soggetto singolo, e la sua volontà è questa volontà singola, riempita del contenuto della singolarità. Ciò significa che l'uomo naturale è egoista. Ma dall'uomo che si dice "buono" esigiamo per lo meno che si orienti secondo determinazioni, leggi universali. La naturalità della volontà è più propriamente l'egoismo della volontà; come volontà naturale la sua volontà è particolare, distinta dall'universalità del volere e contrapposta alla razionalità del volere improntato all'universalità" (G.W.F. Hegel FilRel III, trad. it. 2011, p. 232).

35 "La rappresentazione più comune che si ha della Libertà è quella dell'arbitrio, il quale è termine medio della riflessione posto tra la volontà determinata meramente dagli impulsi naturali e la volontà libera in sé e per sé. [...] L'arbitrio, invece di essere la volontà nella sua Verità, è piuttosto la volontà come contraddizione" (G.W.F. Hegel Dir, trad. it. 1996, p. 103).

${ }^{36}$ Anche in questo caso le opinioni possono discordare. Göschel, per esempio, offre un'interpretazione totalmente diversa da quella che stiamo seguendo: per confermare, forse, la condanna del peccato originale contenuta nelle scritture, egli dimostra come l'arbitrio e di conseguenza anche la trasgressione al divieto divino siano non momenti necessari allo sviluppo della libertà e della coscienza, bensì causa del loro annientamento (Göschel, 1838).

37 "Lo Spirito viene saputo nell'autocoscienza, ed è immediatamente manifesto all'autocoscienza perché coincide con essa: la natura divina è identica alla natura umana, e ciò che qui viene intuito è appunto tale unità" (G.W.F. Hegel Fen, trad. it. 2016, p. 995).

${ }^{38}$ Dal manoscritto: "Questa determinazione [lo spirito naturale] deve essere compresa secondo il suo concetto; essa è immediatamente la contraddizione in se stessa irrisolta" (G.W.F. Hegel FilRel III, trad. it. 2011, p. 54).
} 
spirituale sono qui sinonimi per indicare la vera natura dell'uomo, quella che si scopre a sua volta come libera e che dunque non coincide né con quella immediata dell'egoismo della volontà naturale né con quella scissa dell'arbitrio. ${ }^{39}$

Proprio perché esiste, dunque, una natura "vera" nel senso di razionale e spirituale, sarebbe sbagliato leggere Hegel come un filosofo dell'opposizione natura/spirito o sensi/ragione: la sua impresa mira a conciliare queste scissioni, a salvare in un certo senso la sfera naturale e sensibile attraverso la dimostrazione della loro stessa razionalità, che tuttavia può essere riconosciuta (per sé) solo nell'uomo che ragiona. Si tratta in un certo senso di quel "tornare indietro" che caratterizza l'andamento dialettico, per il quale la determinazione immediata iniziale viene ricompresa come parte del tutto, ovvero parte della ragione e dello spirito.

Tornando al mito qui trattato, mangiare il frutto dell'albero produce quella scissione che fa da elemento mediatore tra l'unità immediata con la natura, che essendo tale sfociava nell'egoismo delle inclinazioni particolari, e l'unità concreta con essa, in cui è avvenuta la conciliazione: il soggetto ha finalmente preso coscienza della razionalità sua propria e del resto della realtà, esercita la cosiddetta volontà libera in sé e per sé. Ciò significa anche, proprio perché si è consapevoli del bene e del male e dell'imputabilità di entrambi, essere in grado di costruire un'etica: l'uomo, infatti, al contrario dell'animale, è educabile di quella Bildung che lo rende un soggetto a tutti gli effetti, ovvero un soggetto autocosciente e libero. ${ }^{40}$ La libertà secondo Hegel sta quindi nell'essere consapevoli dell'essenza di se stessi così come del resto dell'essenza del mondo: si tratta di fatto della medesima sostanza, la ragione, che ci permette di elevarci dalla natura immediata e organizzarci in universalità intersoggettive (anche etiche e politiche) in cui la volontà dei singoli, lungi dall'essere soppressa, si concilia con la volontà universale.

Il mito dell'albero della conoscenza, in conclusione, rappresenta l'uomo che diventa uomo anche perché mostra il passaggio tra le due condizioni di egoismo e interessi particolari da una parte e libertà universale e concreta dall'altra, la quale si qualifica appunto come una conciliazione di particolare ed universale. L'“ermeneutica del concetto"41 che Hegel impiega per leggere l'episodio biblico ha fornito, o meglio svelato, significati profondi riguardanti la

\footnotetext{
39 "L'occupazione dello spirito è quella di prodursi, di farsi oggetto di sé, di sapere di sé; così esso è per se stesso. Le cose della natura non sono per se stesse; perciò non sono libere. Lo spirito produce, realizza se stesso in conformità del suo sapere di sé: esso fa sì che, ciò che esso sa di sé, anche si realizzi” (G.W.F. Hegel FilSt2, trad. it. 1941, pp. 38-39).

${ }^{40}$ M. Antelli, 1990.

${ }^{41}$ G. Garelli, 2015.
} 
coscienza e l'umanità in generale, dando nuova voce al mito e alla rappresentazione religiosa.

\section{Bibliografia primaria}

HEGEL, G.W.F. Enciclopedia delle scienze filosofiche (trad. it. di V. Cicero), Milano: Rusconi, 1996

Fenomenologia dello Spirito (trad. it. di V. Cicero), Milano: Bompiani, 2016

. Lezioni di filosofia della religione (trad. it. di R. Garaventa e S. Achella), voll.I-III, Napoli: Guida, 2003

Lezioni di filosofia della storia (trad. it. Di G. Bonacina e L. Sichirollo) Roma-Bari: Laterza, 2003

. Lezioni sulla filosofia della storia (trad. it. Di G. Calogero e C. Fratta), voll.I-IV, Firenze: La Nuova Italia, 1941

Lineamenti di filosofia del diritto (trad. it. di V. Cicero), Milano: Bompiani 2006

Recensione a Göschel in: Scritti berlinesi (a cura di M. del Vecchio), Milano: Franco Angeli, 2001

Scienza della logica (trad. it. di A. Moni), voll. I-II, Bari: Laterza, 1981

Bibliografia secondaria

ANTELLI, Mauro. Stato di natura e peccato originale in Rousseau ed Hegel in: ACME, vol.43, n.1, Milano: Ledizioni, 1990, pp. 5-16

BORDIGNON, Michela. Il falso non è mica il diavolo. Falso e negativo tra "Prefazione" e "Introduzione" in: Sostanza e soggetto. Studi sulla $<<$ Prefazione $>>$ alla Fenomenologia dello spirito di Hegel (a cura di G. Garelli e M. Pagano), Bologna: Pendragon 2016

CARAMELLI, Eleonora. Lo spirito del ritorno. Studi su concetto e rappresentazione in Hegel, Genova: il melangolo, 2016

CORTELLA, Lucio. L'etica della democrazia. Attualità della Filosofia del diritto di Hegel, Genova-Milano: Marietti, 2011

GARELLI, Gianluca. Dialettica e interpretazione. Studi su Hegel e la metodica del comprendere, Bologna: Pendragon, 2015

GÖSCHEL, Karl Friedrich. Beiträge zur spekulativen Philosophie von Gott und dem Menschen und von dem Gott-Menschen, Berlino: Duncker 1838

HYPPOLITE, Jean. Genesi e struttura della «Fenomenologia dello spirito» di Hegel (trad. it. di G.A. De Toni), Firenze: La Nuova Italia, 1999 
MANCUSO, Vito. Hegel teologo e l'imperdonabile assenza del "Principe di questo mondo", Casale Monferrato: Piemme, 1996

RICEUR, Paul. Le statut de la Vorstellung dans la philosophie hégélienne de la religion In: Qu'est-ce que Dieu? Philosophie/théologie. Hommage à l'abbé Daniel Coppieters de Gibson (1929-1983), Bruxelles: Presses de l’Université Saint-Louis, 1985

RINGLEBEN, Joachim. Hegels Theorie der Sünde. Die subjektivitäts-logische Konstruktion eines theologischen Begriffs, Berlin: Walter de Gruyter GmbH \& Co KG, 1977

Die Dialektik von Freiheit und Sünde: Hegels Interpretation von Gen. 3 in: Biblical Interpretation: History, Context, And Reality, Atlanta: Society of Biblical Lit, 2005

STRAUSS, David Friedrich. La vita di Gesù o esame critico della sua storia (trad. it. di P. Lapertosa e G. Di Iorio), Milano: La Vita Felice 2014

Streitschriften zur Vertheidigung meiner Schrift über das Leben Jesu und zur Charakteristik der gegenwartigen theologie, Tübingen: Osiander, 1837

TRILLHAAS, Wolfgang. Felix culpa. Deutung der Geschichte vom Sündenfall bei Hegel, in Probleme biblischer Theologie in: Probleme biblischer Theologie, München: Kaiser 1971, pp. 589-602

VALENZA, Pierluigi. La circolarità di rappresentazione e concetto nella filosofia della religione di Hegel in: Archivio di filosofia, vol.85, n.1, Pisa: Fabrizio Serra, 2017, pp. 63-77

WOLF, Jean-Claude. "Dass der Mensch durch Erkennen unsterblich ist". Hegel's Deutung der Erzählung vom Sündenfall in: Freiburger Zeitschrift für Philosophie und Theologie, vol.58, n.2, Freiburg: Editions Saint-Paul, 2011, pp. 453-470 